\title{
MEASURABILITY OF FUNCTIONS OF TWO VARIABLES
}

\author{
J. H. MICHAEL and B. C. RENNIE
}

(rec. 30 June 1958)

\section{Summary}

This paper investigates the existence and equality of the double and repeated integrals of a real function on a plane set. The main result (Theorem 2) is that if a function on a plane Lebesgue measurable set is continuous in one variable and measurable in the other then it is measurable in the plane.

\section{Introduction}

Consider the following conditions on a bounded function $f(x, y)$ on the unit square:-

(A) Continuity in $x$ and Lebesgue integrability in $y$.

(B) Riemann integrability in $x$ and in $y$.

(C) Plane L-measurability.

(D) Riemann integrability in $x$ and L-integrability in $y$.

(E) Existence and equality of the two repeated L-integrals.

(F) Existence of the repeated L-integrals.

(G) L-integrability in $x$ and in $y$.

The relations between these conditions are shown in the diagram below, where each condition implies those below it.

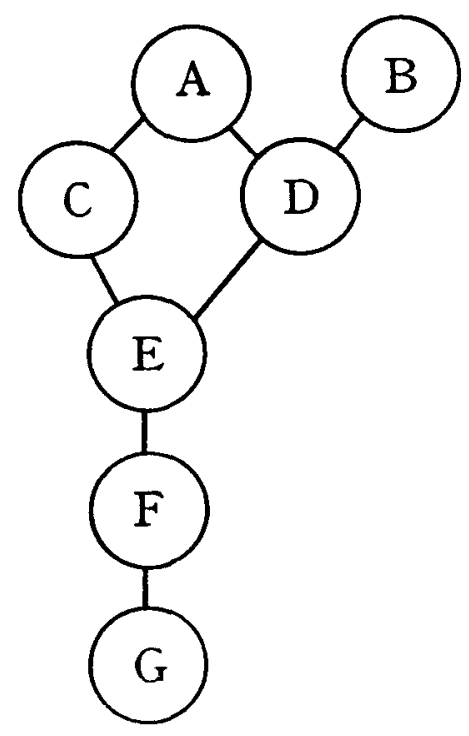


That (A) implies (C) is Theorem 1.

That (C) implies (E) is Fubini's Theorem.

That (D) implies (E) is Theorem 3. The slightly weaker result, that (B) implies (E) was given by L. Lichtenstein [1].

The other implications are trivial.

The problem arises of showing that the diagram above includes all the cases in which one of the seven conditions implies another. This can be done by means of examples given by W. Sierpinski; [2] gives a function satisfying (B) but not (C). It is the characteristic function of a non-measurable set of which any section by a straight line, in any direction, contains at most two points. In [3] he shows how to construct a function satisfying (F) but not $(\mathrm{E})$ or satisfying $(\mathrm{G})$ but not $(\mathrm{F})$. It is easy to construct functions satisfying (C) but not (D) or (D) but not (B), so the completeness of the table of implications is proved.

\section{Notation}

$J$ denotes the unit square $\{(x, y) ; 0 \leqq x \leqq 1,0 \leqq y \leqq 1\}$.

If $A$ is a plane set then for any real $x$ we put:

$$
A_{x *}=\{y ;(x, y) \text { in } A\}
$$

and for any real $y$ we put:

$$
A_{* y}=\{x ;(x, y) \text { in } A\} .
$$

The Lebesgue measure of a measurable set $S$ is denoted by $\mu(S)$, the context should make it clear whether linear or plane measure is meant.

The distance from a point $p$ to a set $S$ is $d[p, S]$.

THEOREM 1. Let $E$ be a closed subset of $J$ and $f$ a real-valued function defined on $E$ and having the following three properties:

i) $0 \leqq f(x, y) \leqq 1$ for all $(x, y)$ in $E$.

(ii) For each real $x$ satisfying $0 \leqq x \leqq 1, f(x, y)$ is measurable on $E_{x *}$ with respect to $y$.

(iii) For each real $y$ satisfying $0 \leqq y \leqq 1, f(x, y)$ is relatively continuous on $E_{* y}$ with respect to $x$.

Then $f$ is plane measurable on $E$.

The truth of this theorem follows from Lemmas 1.1, 1.2 and 1.3, which appear below. In proving these lemmas we assume that $f$ and $E$ are as in the statement of the theorem. We extend the definition of $f$ to be zero on the complement of $E$. We also adopt the following notation.

For each $n=1,2, \cdots$ and $i=1,2, \cdots n$, we can choose a real $a_{n i}$ such that:

$$
(i-1) / n \leqq a_{n i}<i / n
$$

and 
(2)

$$
\mu\left(E_{a_{n i^{*}}}\right) \geqq \underset{(i-1) / n \leqq x<i / n}{1 . \text { u.b. }} \mu\left(E_{x *}\right)-1 / n
$$

We also put:

$$
\begin{gathered}
A_{n i}=\left\{(x, y) ;(i-1) / n \leqq x<i / n,\left(a_{n i}, y\right) \text { in } E\right\} \\
A_{n}=\bigcup_{i=1}^{n} A_{n i} \\
B_{n}=\bigcup_{k=n}^{\infty} A_{k} \\
B=\bigcap_{n=1}^{\infty} B_{n}=\lim \sup A_{k}
\end{gathered}
$$

Evidently each of $A_{n i}, A_{n}, B_{n}$, and $B$ is measurable. By Fubini's theorem we have:

$$
\mu\left(A_{n}\right) \geqq \mu(E)-1 / n \text { for all } n .
$$

For each $n=1,2, \cdots$ and all $(x, y)$ in $J$, define:

$$
\left\{\begin{array}{l}
f_{n}(x, y)=f\left(a_{n i}, y\right) \text { if }(i-1) / n \leqq x<i / n \\
f_{n}(1, y)=0
\end{array}\right.
$$

LEMMA 1.l. $E$ is the union of $B$ with some set of measure zero.

Proof: For each $n=1,2, \cdots$, put:

$$
C_{n}=\{(x, y) ; d[(x, y), E] \leqq 1 / n\}
$$

Since $E$ is closed:

$$
E=\bigcap_{n=1}^{\infty} C_{n}
$$

Also it follows from (3) that if $i \leqq m$ and $n \leqq m$ then $A_{m i} \leqq C_{n}$, and hence by (4) $A_{m} \subseteq C_{n}$, so that by (5):

$$
B_{n} \subseteq C_{n}
$$

By (6), (10) and (11):

$$
B \subseteq E
$$

But by (5) and (7):

$$
\mu\left(B_{n}\right) \geqq \mu(E)-1 / n
$$

Since by (5) $B_{n} \supseteqq B_{n+1}$ we have by (13):

$$
\mu(B)=\lim \mu\left(B_{n}\right) \geqq \mu(E)
$$

Therefore by (12) and (14) the result follows.

LEMMA 1.2. For each $n=1,2, \cdots$ the function $f_{n}(x, y)$ is plane measurable on $J$ and hence also on $E$.

Proof: For each $i=1,2 \cdots n$, put: 


$$
J_{i}=\{(x, y) ;(i-1) / n \leqq x<i / n, 0 \leqq y \leqq 1\}
$$

Then by (8):

$$
f_{n}(x, y)=f\left(a_{n i}, y\right) \text { for all }(x, y) \text { in } J_{i}
$$

But by condition (ii) of the theorem, $f\left(a_{n i}, y\right)$ is measurable with respect to $y$ on the unit interval. Therefore by $(15) f_{n}(x, y)$ is plane measurable on $J_{i}$ and hence on $J$.

LEMMA 1.3. For each $(x, y)$ in $B$ :

$$
\lim \sup _{n}(x, y)=f(x, y)
$$

Proof: Take any $(x, y)$ in $B$, we know $x \neq 1$.

For each positive integer $k$ there is $i$ such that $(i-1) / k \leqq x<i / k$. First consider the set of all $k$ for which $(x, y)$ is in $A_{k}$, it is an infinite set because by (6) the set $B$ is the upper limit of the sequence $A_{1}, A_{2}, \cdots$

Take any $\varepsilon>0$.

For all sufficiently large $k$ it follows from the condition (iii) of the theorem that

$$
|f(\xi, y)-f(x, y)|<\varepsilon
$$

for all $\xi$ such that $(\xi, y)$ is in $E$ and $|x-\xi|<1 / k$.

In particular $a_{k i}$ satisfies these conditions for $\xi$, so that

i.e.

$$
\left|f\left(a_{k i}, y\right)-f(x, y)\right|<\varepsilon
$$

$$
\left|f_{k}(x, y)-f(x, y)\right|<\varepsilon
$$

This holds for all $\varepsilon>0$, so we have shown that as $k$ tends to infinity in this subset (the subset for which $(x, y)$ is in $A_{k}$ )

$$
f_{k}(x, y) \rightarrow f(x, y) \text {. }
$$

Now consider the other values of $k$, for which $(x, y)$ is not in $A_{k}$. For these values $\left(a_{k i}, y\right)$ is not in $E$ by (3) and (4), and therefore $f_{k}(x, y)=$ $f\left(a_{k i}, y\right)=0$.

We have thus shown that the sequence $f_{k}(x, y)(k=1,2, \cdots)$ contains an infinite sub-sequence converging to $f(x, y)$ and that the members not in this sub-sequence are all zero.

The measurability of $f(x, y)$ on $B$ and therefore on $E$ follows from the three lemmas.

THEOREM 2. Suppose that $f(x, y)$ is defined on a measurable plane set $E$ and is 0 outside $E$ and that $f(x, y)$ is a continuous function of $x$ on $E$ relatively to $E$ for almost all $y$ and that it is a measurable function of $y$ for almost all $x$; then $f(x, y)$ is plane measurable.

Proof: By Theorem 1 the result holds when the following four additional conditions are satisfied: 
(a) $E$ is closed,

(B) $E$ is a subset of the unit square and $f \leqq 1$,

$(\gamma) f \geqq 0$,

( $\delta$ ) In the statement of the theorem 'almost all' may be replaced by 'all'.

These restrictions can be removed one by one as follows:

(a) There is a sequence of closed sets $E_{n}$ increasing and converging in measure to $E$. Let $f_{n}(x, y)$ be equal to $f$ if $(x, y)$ is in $E_{n}$ and 0 otherwise. These functions are all plane measurable and converge to $f(x, y)$ almost everywhere.

( $\beta$ ) Let $f_{n}(x, y)$ be 0 if $|x|$ or $|y|>n$ and otherwise let it be the smaller of $f$ and $n$, then each $f_{n}$ is plane measurable and therefore also $f$.

$(\gamma)$ Apply the results already obtained to the positive and negative parts of $f$.

( $\delta)$ The set of points affected is of plane measure zero, so they can be removed from $E$.

Some more definitions are required for the next theorem:

A 'Riemann dissection' of the unit interval is a choice of real $a_{n}$ and $c_{n}$ for $n=1,2, \cdots N$ such that $a_{N}=1$ and (if $a_{0}=0$ ) $a_{n-1} \leqq c_{n} \leqq a_{n}$ for $n=1,2, \cdots N$. The 'Riemann sum' for this dissection and a function $f(x)$ is $\Sigma\left(a_{n}-a_{n-1}\right) f\left(c_{n}\right)$.

If $\mathscr{D}$ is such a dissection let $\Delta(\mathscr{D})=\max \left(a_{n}-a_{n-1}\right)$.

We say that a sequence $\mathscr{D}_{1}, \mathscr{D}_{2}, \cdots$ of Riemann dissections 'converges' if $\Delta\left(\mathscr{D}_{n}\right) \rightarrow 0$ as $n \rightarrow \infty$.

It can be shown that a function is Riemann integrable if and only if for any convergent sequence of Riemann dissections the Riemann sums converge, and that if so then the limit equals the integral.

THEOREM 3. If in the unit square $J$ the function $f(x, y)$ is Riemann integrable in $x$ for each $y$ and Lebesgue measurable in $y$ for each $x$, and $0 \leqq t \leqq 1$, then the repeated integrals exist and are equal.

Proof. Let $\mathscr{D}_{1}, \mathscr{D}_{2}, \cdots$ be any convergent sequence of Riemann dissections of the unit interval.

Let $g_{n}(y)$ be the Riemann sum of $f(x, y)$ with respect to $x$ corresponding to the dissection $\mathscr{D}_{n}$. The function $g_{n}(y)$ is measurable in $y$ because it is the sum of a finite number of measurable functions, so that its limit, which is $\int_{0}^{1} f d x$, is also a measurable function of $y$.

The Riemann sum of $\int_{0}^{1} f d y$ corresponding to $\mathscr{D}_{n}$ is $\int g_{n}(y) d y$, which by the theorem of bounded convergence tends to

$$
\int_{0}^{1}\left(\int_{0}^{1} f d x\right) d y
$$

as $n$ tends to infinity. This is for any convergent sequence of Riemann 
dissections, so that the Riemann integral of $\int_{0}^{1} f d y$ exsists and equals $\int_{0}^{1}\left(\int_{0}^{1} f d x\right) d y$.

\section{References}

[1] Lichtenstein, L., Prace matematyczno-fizyczne, T. XXI, 11.

[2] Sierpinski, W., 'Sur un probleme concernant les ensembles mesurables superficiellement', Fund. Math., I (1920), 112-115.

[3] Sierpinski, W., 'Sur les rapports entre l'existence des integrales:

$$
\int_{0}^{1} f(x, y) d x, \quad \int_{0}^{1} f(x, y) d y \text { et } \int_{0}^{1} d x \int_{0}^{1} f(x, y) d y .
$$

Fund. Math. I (1920), 142-147.

University of Adelaide 